\title{
Diets rich in n-6 PUFA induce intestinal microbial dysbiosis in aged mice
}

\author{
Sanjoy Ghosht, Erin Molcant, Daniella DeCoffe, Chaunbin Dai and Deanna L. Gibson* \\ ASC 368, 3333 University Way, Department of Biology, The Irving K. Barber School of Arts and Sciences, University of British \\ Columbia Okanagan, Kelowna, BC, Canada V1V 1V7
}

(Submitted 19 July 2012 - Final revision received 8 October 2012 - Accepted 29 October 2012 - First published online 8 January 2013)

\begin{abstract}
Controversies have emerged regarding the beneficial $v$. detrimental effects of dietary $n-6$ PUFA. The alteration of the intestinal microbiota, a phenomenon termed dysbiosis, occurs during several chronic inflammatory diseases, but has not been well studied in an aged population. With present 'Western' diets predominantly composed of $n-6$ PUFA, we hypothesised that PUFA-rich diets cause intestinal dysbiosis in an aged population. C57BL/6 mice (aged 2 years) were fed a high-fat (40\% energy), isoenergetic and isonitrogenous diet composed of rapeseed oil, maize oil or maize oil supplemented with fish oil. We examined ileal microbiota using fluorescence in situ hybridisation and stained tissues by immunofluorescence for the presence of immune cells and oxidative stress. We observed that feeding high-fat diets rich in $n-6$ PUFA promoted bacterial overgrowth but depleted microbes from the Bacteroidetes and Firmicutes phyla. This corresponded with increased body mass and infiltration of macrophages and neutrophils. Fish oil supplementation (rich in long-chain $n$-3 PUFA like DHA and EPA) restored the microbiota and inflammatory cell infiltration and promoted regulatory T-cell recruitment. However, fish oil supplementation was associated with increased oxidative stress, evident by the increased presence of 4-hydroxynonenal, a product of lipid peroxidation. These results suggest that an $n-6$ PUFA-rich diet can cause dysbiosis and intestinal inflammation in aged mice. However, while fish oil supplementation on an $n-6$ PUFA diet reverses dysbiosis, the combination of $n-6$ and $n-3$ PUFA, like DHA/EPA, leads to increased oxidative stress, which could exacerbate gastrointestinal disorders in the elderly.
\end{abstract}

Key words: PUFA: Ageing: Intestinal microbiota: Dysbiosis: Intestinal inflammation

The microbiota is an intricate system of micro-organisms consisting of over a thousand different species of commensal bacteria, including a small amount of potentially pathogenic bacteria surviving in a symbiotic relationship with its host ${ }^{(1)}$. The human gut microbiota mainly consists of two bacterial phylogenies: Bacteroidetes $(\mathrm{CFB})$ and Firmicutes $(\mathrm{FIRM})^{(2)}$. A smaller amount of bacteria is represented by the Proteobacteria (GAM) and Actinobacteria phyla ${ }^{(3)}$. Particular interest lies in the FIRM and CFB phyla ${ }^{(4)}$, as many members are considered beneficial to the alimentary system, contributing significantly to the functionality of the intestine. Often, in individuals experiencing dysbiosis, such as that during obesity, the ratio of FIRM:CFB changes ${ }^{(5)}$. An alteration in the ratio of these phyla is also known to occur in inflammatory bowel disease (IBD) ${ }^{(6)}$, ageing $^{(7)}$, diabetes ${ }^{(8,9)}$ and the metabolic syndrome ${ }^{(10)}$. Disruption of these bacterial populations may have detrimental effects on the immunological and protective functions that occur in the host.
While host genetics has some control over the composition of the microbiota ${ }^{(11)}$, diet as an extrinsic factor has received much attention recently. Over the past 30 years, industrialised countries have shifted their consumption of fats from that of saturated fats, to predominantly n-6 PUFA from vegetable oils, such as maize, safflower and sunflower oils ${ }^{(12)}$. More interestingly, high $n$ - 6 PUFA induces a $30 \%$ increase in the risk of ulcerative colitis, whereas $n$ - 3 PUFA may decrease the incidence of colitis by upto $77 \%{ }^{(13)}$. A high- $n-6$, low- $n-3$ PUFA diet has been linked to several other pro-inflammatory conditions such as insulin resistance ${ }^{(14,15)}$, atherosclerosis $^{(16,17)}$, colorectal ${ }^{(18,19)}$ and pancreatic cancers ${ }^{(20)}$ and $\mathrm{IBD}^{(21,22)}$. Although likely, it is yet unknown if the microbiota and the intestinal inflammatory milieu are altered with such diets.

Ageing has been recently associated with microbial dysbiosis ${ }^{(23)}$, and at different stages in life the ratio of FIRM:CFB has been shown to be different ${ }^{(7)}$. The microbial population in ageing human subjects shows a rearrangement in the FIRM

Abbreviations: 4-HNE, 4-hydroxynonenal; CFB, Bacteroidetes; FIRM, Firmicutes; GAM, Proteobacteria; IBD, inflammatory bowel disease; $\mathrm{T}_{\text {reg, }}$, regulatory T.

*Corresponding author: D. L. Gibson, fax +1 250807 8000, email deanna.gibson@ubc.ca

† S. Ghosh and E. Molcan are co-first authors. 
population and a higher abundance of facultative anaerobic bacteria associated with increased inflammation ${ }^{(24)}$. Both intestinal diseases and inflammation progress with age, but it is unknown if diet can affect microbiota compositions in an aged population, thereby promoting altered host responses such as inflammation.

The present study investigated the effect of diets rich in various PUFA on dysbiosis, immune cell infiltration and oxidative stress in the small-intestinal ileum of aged mice. We found that diets rich in maize or rapeseed oil rich in linoleic acid (called n-6 PUFA) promoted bacterial overgrowth, but depleted microbes from the major gut phyla, CFB and FIRM. This corresponded to an increase in body mass and infiltration of macrophages and neutrophils into the ileal submucosae. In contrast, fish oil-supplemented diets, rich in EPA and DHA, restored microbial numbers and composition, recruited regulatory $\mathrm{T}\left(\mathrm{T}_{\text {reg }}\right)$ cells and decreased the infiltration of macrophages and neutrophils, but were associated with increased oxidative stress, evident by increased immunolocalisation of 4-hydroxynonenal (4-HNE), a product of lipid peroxidation. These results suggest that, while fish oil supplementation can reverse detrimental effects of excess $n$ - 6 PUFA, the present study also demonstrates that the combination of $n-6$ PUFA and DHA/EPA could lead to unintended consequences, such as oxidative stress, which have the potential to lead to increased susceptibility to intestinal dysfunction in an aged population.

\section{Experimental methods}

\section{Mice and feeding protocols}

Female C57BL/ 6 mice (aged 24 months) were maintained from birth at the Child and Family Research Institute at the University of British Columbia. Mice were fed $\gamma$-irradiated food and tap water under specific pathogen-free conditions in animal facilities. The protocols used were approved by the University of British Columbia's Animal Care Committee and are in direct accordance with the guidelines drafted by the Canadian Council on the Use of Laboratory Animals.

Mice were fed a high-fat ( $40 \%$ energy) or a 'normal' chow ( $9 \%$ energy from fat) diet for $33 \mathrm{~d}$. In total, $40 \%$ energy represents the upper limit of human fat consumption ${ }^{(25)}$. Earlier studies have demonstrated that, akin to the present study, feeding $20 \%(\mathrm{w} / \mathrm{w})$ sunflower oil for 4 weeks increases the n-6 PUFA content of circulating lipoproteins, a major source of fatty acids to the host tissues ${ }^{(26)}$. The high-fat diets were isoenergetic and isonitrogenous, and were prepared by adding $200 \mathrm{~g}$ of oil to $800 \mathrm{~g}$ of basal mix obtained from Harlan Teklad (catalogue no. TD.88.232). The oils used were $20 \%$ $(\mathrm{w} / \mathrm{w})$ rapeseed oil (low- $n-6$ group), $20 \%(\mathrm{w} / \mathrm{w})$ maize oil (high- $n-6$ group) or $19 \%(\mathrm{w} / \mathrm{w})$ maize oil supplemented with $1 \%(\mathrm{w} / \mathrm{w})$ fish oil (high- $n-6+$ long-chain $n-3$ PUFA group). The fatty acid compositions of the various oils are given in Table 1, while the energy and macronutrient composition of the basal mix is listed in Table 2 .

For fish oil-supplemented diets, we followed the guidelines set by the American Heart Association, which recommends 0.5-1.8g of long-chain EPA + DHA to offset high levels of
Table 1. Major fatty acid compositions of dietary oil used in preparing high-fat diets*

\begin{tabular}{lccc}
\hline Lipids & Maize oil & Rapeseed oil & Fish oil \\
\hline SFA & 2.56 & 1.38 & 2.75 \\
Linoleic acid $(n-6)$ & 11.46 & 3.88 & 10.9 \\
Arachidonic acid & 0 & 0 & 0.025 \\
$\alpha$-Linolenic acid $(n-3)$ & 0.24 & 1.5 & 0.231 \\
Oleic acid & 5.36 & 12.92 & 5.31 \\
DHA and EPA $(n-3)$ & 0 & 0 & 0.34 \\
\hline
\end{tabular}

${ }^{*}$ Lipids are listed as $\mathrm{g} / 100 \mathrm{~g}$ of diet.

n-6 PUFA, like linoleic acid (average intakes of $18 \mathrm{~g} / \mathrm{d}$ in USA), and treat cardiovascular disorders ${ }^{(27,28)}$. Therefore, a ratio of 20:1 (w/w) of oils is required to mimic human dosing with the lowest dose of fish oil. As depicted in Table 1, EPA + DHA constitute $34 \%$ of fish oil by weight, and therefore, $0.5 \mathrm{~g}$ of $\mathrm{EPA}+\mathrm{DHA}$ is available in $1.5 \mathrm{~g}$ of fish oil. Similarly, $18 \mathrm{~g}$ of linoleic acid is present in $30 \mathrm{~g}$ of maize oil. Therefore, we added $1 \%(\mathrm{w} / \mathrm{w})$ fish oil with $19 \%(\mathrm{w} / \mathrm{w})$ maize oil for the fish oil-supplemented diet. A high-fat diet with fish oil was exclusively avoided as higher percentages of fish oil $(8 \%(\mathrm{w} / \mathrm{w})$ and beyond) are not only supraphysiological but can induce oxidative stress and inflammation per se (29,30) $^{2}$

\section{Tissue collection}

After the feeding protocols were completed, the mice were killed and small-intestinal ileal tissue samples were collected. Adherent microbes were separated from the ileal tissues through homogenisation and fixed in $3.7 \%$ formalin for analysis. For immunofluorescence, ileal tissues were fixed in $3.7 \%$ formalin, embedded in paraffin and $3 \mu \mathrm{m}$ intestinal sections were sectioned by the histology group at the Child and Family Research Institute at the University of British Columbia.

\section{Microbiota analysis}

To examine ileal microbial ecology, fluorescence in situ hybridisation was used similar to Ghosh et al. ${ }^{(31)}$. Briefly, probes used to hybridise microbiota samples were Eubacteria (EUB) (FarRed-conjugated EUB338 general bacterial probe; EUB338: 5'-GCTGCCTCCCGTAGGAGT-3'), CFB (Texas redconjugated CFB probe; CFB286: 5'-TCCTCTCAGAACCCCTAC-

Table 2. Diet composition of the basal mix (Harlan Teklad; TD.88232) used in all diets*

\begin{tabular}{lr} 
Formula & \\
\hline Casein & $300 \cdot 0$ \\
DL-Met & 4.5 \\
Sucrose & 373.5 \\
Maize starch & 187.5 \\
Cellulose & 62.5 \\
Mineral mix, AIN-76 170915 & 52.5 \\
Calcium carbonate & 4.5 \\
Vitamin mix (Teklad 40060) & 15.0 \\
\hline
\end{tabular}

${ }^{*}$ Each diet contained $13.81 \mathrm{~kJ} / \mathrm{g}(3.3 \mathrm{kcal} / \mathrm{g})$ of energy composed of protein at $26.6 \%$ by weight, carbohydrate at $55.5 \%$ by weight and fat at $0.3 \%$ by weight. 
3'), FIRM (AlexaFluor-conjugated FIRM probes: LGC354a: 5'-TGGAAGATTCCCTACTGC-3'; LGC354b: 5'-CGGAAGATTCCCTACTGC- $3^{\prime}$; LGC354C: $5^{\prime}$-CCGAAGATTCCCTACTGC- $3^{\prime}$ ) and $\gamma$-GAM (AlexaFluor-conjugated GAM probe; GAM42a: 5'-GCCTTCCCACATCGTTT- $3^{\prime}$ ). Sequence specificity of $\mathrm{CFB}$ and $\mathrm{GAM}^{(32)}$, as well as $\mathrm{EUB}^{(32)}$ and $\mathrm{LGC}^{(33)}$, probes was confirmed through the 16S RNA Ribosomal Database or the Probe Check Database (http://131.130.66.200/cgi-bin/probecheck/ content.pl?id=home). Probes were hybridised to bacteria filtered onto Nucleopore polycarbonate membranes and imaged using a fluorescent microscope. Percentages of CFB, GAM, FIRM and all other bacteria were calculated based on the number of positively probed bacteria, visualised in five to six randomly selected fields of view, against the number of positively probed Eubacteria representing total bacteria. For quantifying total microbial loads, SYBR green staining (nucleic acid stain; Invitrogen) was used on bacterial samples. The samples were filtered onto Anodisc 25 membranes, stained, mounted and imaged as mentioned earlier. Total microbiota load was calculated by averaging the number of SYBR-positive cells/g of tissue weight, accounting for a total filter area of $2.01 \mathrm{~cm}^{2}$, picture dimensions of $5.622 \times 10^{-2} \mathrm{~cm}^{2}$ and $100 \times$ objective magnification. To determine if the bacteria were interacting with the host epithelium, fluorescence in situ hybridization was performed on tissue sections by deparaffinising and rehydrating paraffin-embedded tissue sections and incubating overnight at $37^{\circ} \mathrm{C}$ in the dark with probes. Sections were washed with hybridisation solution, wash buffer and deionised water. Samples were then mounted using fluoroshield with 4',6-diamidino-2-phenylindole (DAPI) (Sigma) and viewed using an Olympus IX81 fluorescent microscope.

\section{Immunofluorescence}

Paraffin-embedded tissue sections were deparaffinised and rehydrated using standard techniques. Antigen retrieval of rehydrated tissues was performed using a $1 \mathrm{mg} / \mathrm{ml}$ trypsin (Sigma) for 20-30 min at room temperature. The tissue was washed and blocked with 5\% bovine serum albumin (Sigma) followed by incubation with the following primary antibodies: myeloperoxidase rabbit polyclonal antibody-1 (Thermo Scientific), to examine neutrophils; F4/80 mouse monoclonal antibody (CedarLane), to examine macrophages; FOXP3 (H-190) rabbit polyclonal antibody IgG (Santa Cruz Biotechnology), to examine $\mathrm{T}_{\text {reg }}$ cells and anti-4-HNE goat polyclonal antibody (Abcam) followed by goat anti-rabbit IgG AlexaFluorconjugated 594-red antibody (Invitrogen), goat anti-rabbit IgG 488-conjugated antibody (Rockland), goat anti-mouse IgG HiLyteFluor 488-labelled antibody or rabbit anti-goat 594-labelled IgG (Ana Spec, Inc.). Tissue sections were mounted and viewed as mentioned earlier. For inflammatory cell counts, positive cells were identified by blinded observers and quantified. For 4-HNE quantification, fluorescence intensity was measured using MetaMorph ${ }^{\circledR}$ software for Olympus, http://support.meta.moleculardevices.com/docs/mm\%20bag. pdf, compatible with an Olympus IX81 microscope (Molecular Devices, Inc.).

\section{Statistical analysis}

The results are expressed as mean values with standard errors and graphed in GraphPad Prism version 4.00 for Windows (GraphPad Software). Statistical analysis was performed using a non-parametric Mann-Whitney $t$ test at a 95\% CI ( $n$ 5-6 mice in each diet group).

\section{Results}

n-6 PUFA-rich diets caused bacterial overgrowth and dysbiosis associated with weight gain and dietary n-3 PUFA reversed these effects

C57BL/6 mice (aged 2 years) were fed a high-fat (40\% energy), isoenergetic and isonitrogenous diet composed of maize oil (high $n$-6 PUFA), maize oil supplemented with fish oil (high n-6 PUFA supplemented with long-chain $n$-3 PUFA) or rapeseed oil (low $n-6$ PUFA) for $33 \mathrm{~d}$. To determine the effect of dietary PUFA on the intestinal microbiota, we examined the microbes associated with the ileal samples obtained from the groups of mice. Since bacterial overgrowth has been associated with excessive inflammation in the gut ${ }^{(34)}$, we examined total bacterial loads and found that the maize oil diet causes bacterial overgrowth (Fig. 1(a)). In contrast, animals given fish oil supplementation reversed ileal bacterial overgrowth to levels observed with normal chow.

To determine the effect of dietary PUFA on dysbiosis, we examined bacteria from major gut phyla CFB and FIRM, as well as GAM, using fluorescent in situ hybridisation. Compared with the normal chow, both the maize and rapeseed oil-fed groups showed the greatest changes in bacterial composition (Fig. 1(b)). The maize oil-fed group had significantly fewer bacteria detected from the FIRM and CFB phyla compared with normal chow, whereas the rapeseed oil-fed group had significantly fewer bacteria from the CFB phylum. This resulted in an increased ratio of FIRM:CFB for both of the maize and rapeseed oil-fed groups compared with the normal chow (Fig. 1(c)). This change in ratio has been associated with various disease profiles including obesity ${ }^{(10,35)}$, and indeed, mice fed maize or rapeseed oil had significantly increased body mass compared with the normal chow groups (Fig. 2). However, such changes could not be attributed to higher energy density per se, as fish oil supplementation had significantly less weight gain compared with both maize and rapeseed oil groups. Instead, we hypothesise that the reduced weight gain was due to the restored bacterial composition, as the FIRM:CFB ratio was similar to that of the normal chow control group in the fish oil-supplemented group.

\section{All high-fat diets resulted in a close association of the microbiota to the intestinal mucosal surface, but only the n-6 PUFA-rich diet resulted in bacterial infiltration across the intestinal barrier}

In a healthy gut, it is thought that the bacteria are separated from the intestinal mucosal surface by mucin layers. To examine if dietary PUFA influenced the location of the microbiota in the ileum, we examined the FIRM and CFB bacteria using 
(a)

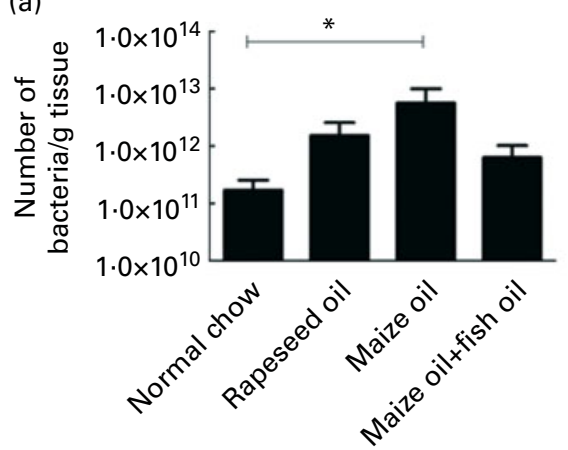

(b)

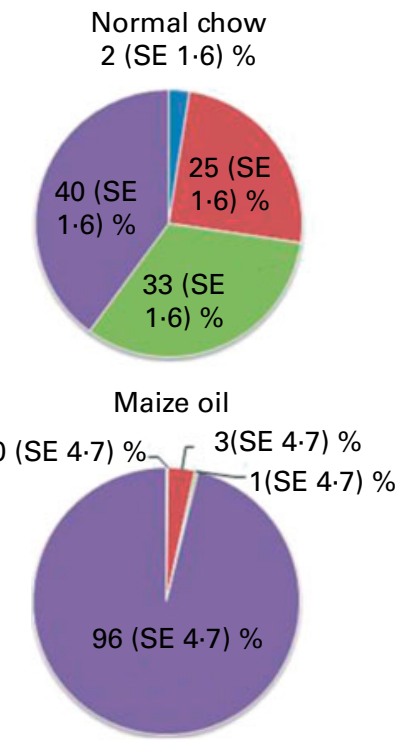

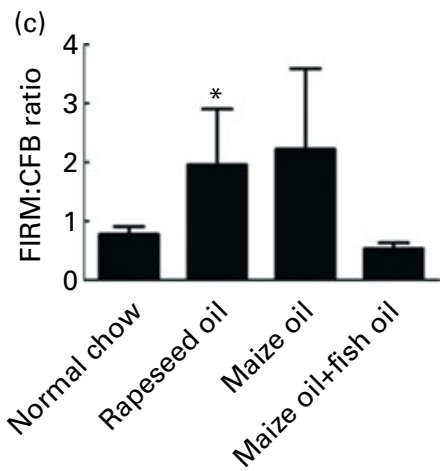

Rapeseed oil 0 (SE 3.8) \%



Maize oil+fish oil 1 (SE $2 \cdot 0) \%$

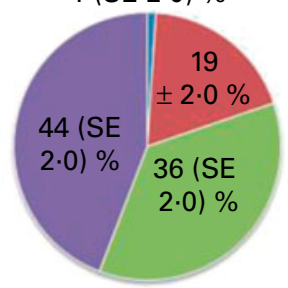

Fig. 1. Maize oil diets rich in $n-6$ PUFA cause bacterial overgrowth and dysbiosis in aged mice, while fish oil supplementation rich in $n$-3 PUFA can reverse these effects. (a) Total number of bacteria per $\mathrm{g}$ of tissue in the ileum quantified using SYBR green nucleic acid staining. Mice fed maize oil diets had significantly more bacteria compared with the normal chow controls $\left({ }^{*} P<0.05\right)$, whereas fish oil supplementation had similar levels compared with the normal chow controls. (b) The relative abundance of different bacterial phyla with their standard errors in the ileum was determined via fluorescence in situ hybridisation using specific probes to the bacterial phyla Bacteroidetes (CFB, $\square$ ), Firmicutes (FIRM, $\square$ ) and $\gamma$-Proteobacteria (GAM, $\square$ ). All other bacteria were counted as unknown ( $\square$ ). (c) The ratio of FIRM:CFB was calculated based on fluorescence in situ hybridisation percentages. The maize and rapeseed oil-fed groups have a higher ratio than normal chow controls, whereas the fish oil supplementation group has a similar ratio as the normal chow control group.

specific probes on tissues sections to highlight the bacterial interaction with the intestinal mucosal surface (Fig. 3). While we found that the microbiota was not in close association with the intestinal mucosal surface in the normal chow group, the bacteria were closely associated with the intestinal epithelia in all the high-fat diet groups. However, the maize oil group showed increased bacterial infiltration into the intestinal epithelium (Fig. 3; white arrows). Notably, fish oil supplementation resulted in decreased bacterial infiltration.

\section{Maize oil diets increased infiltration of neutrophils and macrophages and fish oil supplementation recruited immunosuppressive regulatory $T$ cells}

Since bacterial infiltration across the mucosal surface could increase inflammatory responses, we examined neutrophil, macrophage and $\mathrm{T}_{\text {reg }}$ cells infiltration on tissue sections by immunofluorescence (Fig. 4). Maize oil, similar to rapeseed oil diets, resulted in the infiltration of $\mathrm{F} 4 / 80+$ macrophages and myeloperoxidase + neutrophils, but only a few $\mathrm{T}_{\text {reg }}$ cells, which is consistent with increased intestinal inflammation. In contrast, supplementation with fish oil restored inflammatory cell infiltration similar to normal chow controls and increased $\mathrm{T}_{\text {reg }}$ cell recruitment beyond normal chow levels (Fig.4(e)). This suggests that fish oil may have the capacity to decrease the level of inflammation associated with high-fat feeding in aged mice.

\section{Supplementing maize oil diets with fish oil increased 4-hydroxynonenal in the ileum}

Since oxidative stress has been associated with ageing and PUFA consumption, we investigated the abundance of 4-HNE, a product of lipid peroxidation (Fig. 5). Surprisingly, the presence of 4-HNE was highest in the dietary fish oil group, mostly present in the serosal side of the submucosal 


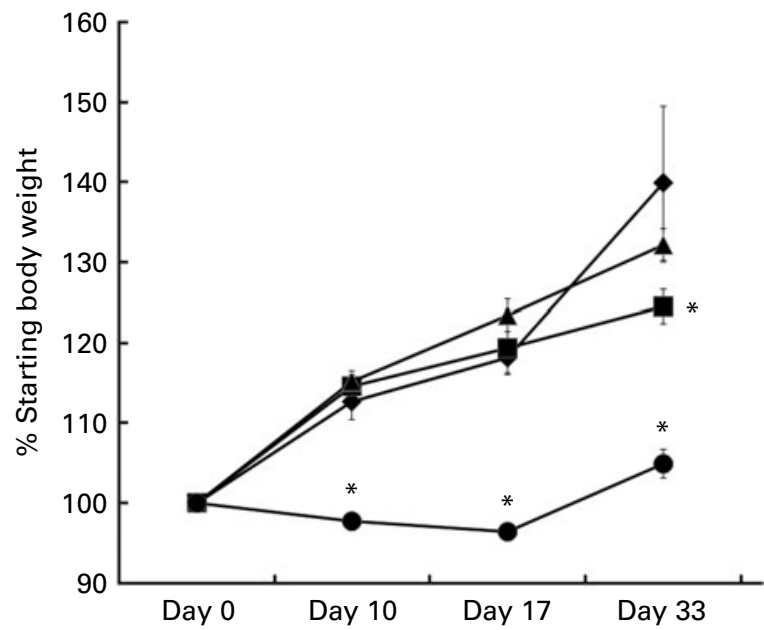

Fig. 2. Maize and rapeseed oil diets fed to aged mice result in significant increases in body weight, whereas fish oil supplementation was associated with reduced weight gain. Body weight was measured at the indicated days after feeding high-fat diets and was calculated as the percentage of starting body weight at each time point. While all high-fat diets showed significantly more weight gain compared with the normal chow group, the fish oil-supplemented group showed significantly less weight gain at day 33 compared with both maize and rapeseed oil diets $\left({ }^{*} P<0.05\right)$. $\multimap$, Maize oil; $-\mathbf{-}-$, maize oil + fish oil; - $-\mathbf{-}$, rapeseed oil; - $\bullet-$, normal chow.

region of the ileum. Since oxidative stress can damage host cellular components such that the functionality of the cell is reduced, this may be a negative consequence associated with DHA + EPA supplementation on an already high- $n-6$ PUFA diet.

\section{Discussion}

The relationship between diet and health is complex when considering the fact that dietary antigens interact with both the gastrointestinal microflora as well as the host mucosal surfaces. This is further complicated by the fact that all dietary oils are a mixture of various fatty acids, which makes attribution of any biological effects to a single type of fatty acid difficult. The intestinal microbiota plays a crucial role in early postnatal development of immunity, but the microbiota and ensuing immunological responses have not been well studied in an aged population. This is a critical group to study, considering that many chronic diseases while diagnosed in early adulthood persist into old age. The recent rise in rates of inflammatory diseases suggests a major contribution from changing environmental determinants like diet.

In the last few decades, there has been a shift in the amount and composition of fats associated with a 'Western' diet. In Canada, as an example, over the last 30 years, the consumption of $n-6$ PUFA increased by over $54 \%$, as saturated fat consumption has plateaued ${ }^{(36)}$. In the present study, we examined the effect of dietary PUFA in the gastrointestinal tract of aged mice to determine the effect on microbial ecology. We found that maize and rapeseed oil diets containing $n$-6 PUFA promoted dysbiosis, or ecological disturbances to the intestinal microbiota, which was associated with increased infiltration of macrophages and neutrophils. While initially we hypothesised that either the low $n-6$ PUFA or the high $n-3$ PUFA, like $\alpha$-linolenic acid, would be protective against dysbiosis, the lack of protection with rapeseed oil was intriguing. Therefore, with an abundance of $n-6$ PUFA, $\alpha$-linolenic acid may not provide benefit or may not undergo sufficient bioconversion to its long-chain metabolites $\mathrm{DHA}+\mathrm{EPA}^{(37)}$. In contrast, fish oil supplementation, which directly added DHA + EPA, restored the microbiota as well as inflammatory responses compared with the normal chow-fed mice. However, fish oil supplementation was also associated with increased 4-HNE, a product of lipid peroxidation. The present study reveals that, while fish oil supplementation can reverse the effects of $n-6$ PUFA diets on intestinal microbial dysbiosis and inflammatory cell infiltration, the oxidative stress response may increase as an unintended consequence.

Dysbiosis, or altered microbes, has been observed in individuals with many chronic inflammatory diseases like $\operatorname{IBD}^{(6)}$, diabetes ${ }^{(8,9)}$, the metabolic syndrome ${ }^{(10)}$ and obesity ${ }^{(5)}$ Ageing has been recently associated with microbial dysbiosis ${ }^{(23)}$, resulting in a rearrangement of the FIRM population associated with increased inflammation ${ }^{(24)}$. The cause of dysbiosis is relatively unknown. In the present study, we show that various fat diets have different effects on the composition of the intestinal microbiota in aged mice. High-fat diets rich in maize or rapeseed oil resulted in an increase in the FIRM:CFB ratio. In contrast, fish oil supplementation reversed this dysbiosis, since the microbial composition was
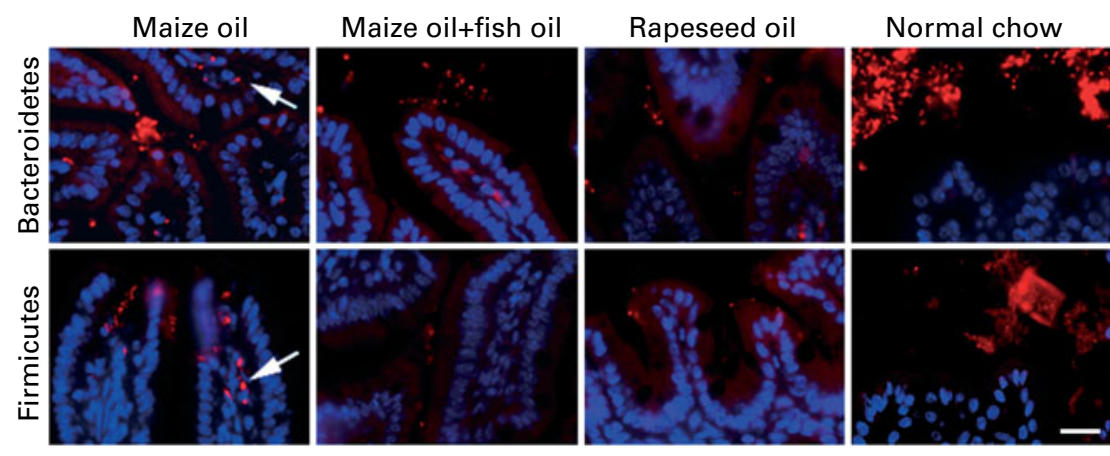

Fig. 3. While all high-fat diets result in a close association of the microbiota with the intestinal mucosal surface, maize oil diets result in bacterial infiltration across the intestinal barrier. Using fluorescence in situ hybridisation and specific probes to Bacteroidetes and Firmicutes on tissue sections of ileum samples, we found that all high-fat diets resulted in bacteria (stained in red) in close association with the intestinal epithelia (host nuclei stained with DAPI in blue). Only the maize oil diet group showed bacterial infiltration into the intestinal epithelium $(\Leftrightarrow$; bar $=200 \mu \mathrm{m} ; 630 \times$ magnification $)$. 



Rapeseed oil

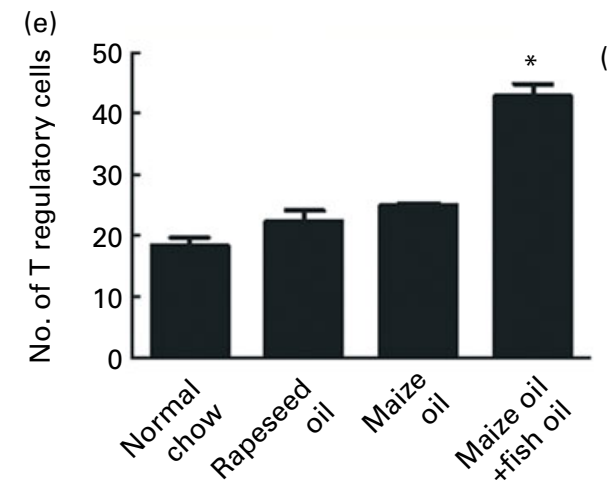

Maize oil

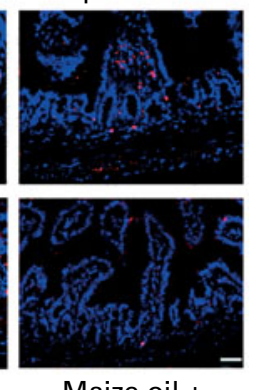

Maize oil +

fish oil

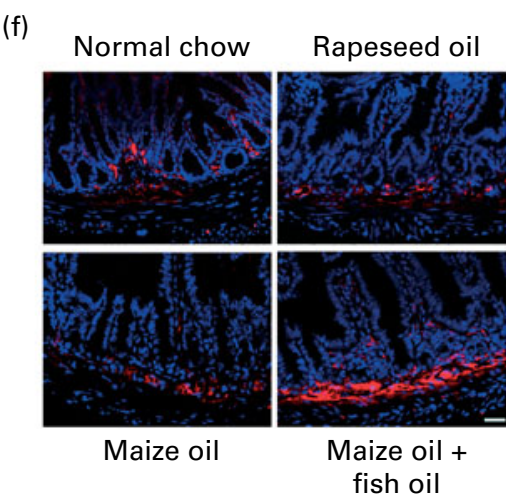

Fig. 4. While maize and rapeseed oil diets increase infiltration of neutrophils and macrophages, fish oil supplementation significantly decreases their infiltration and recruits immunosuppressive regulatory T cells. (a), (c) and (e) Using immunofluorescence and antibodies specific to either F4/80 (macrophages), MPO (neutrophils) or FoxP3 regulatory T cells, we quantified the presence of immune cells per ileal section from mice fed the various diets ( $\left.{ }^{*} P<0 \cdot 05,{ }^{* \star} P<0 \cdot 005\right)$. (b), (d) and (f) show representative images of ileal sections stained for F4/80 (green), MPO (red) or FoxP3 (red), where host cell nuclei are stained with 4',6-diamidino-2-phenylindale (DAPI) (blue) (bar $=100 \mu \mathrm{m} ; 200 \times$ magnification).

similar to the normal chow control diet. The increase in FIRM has been associated with obese individuals ${ }^{(5)}$ and an increased capability of harvesting energy from the intestine ${ }^{(38)}$. Similarly, we found that maize and rapeseed oil-fed mice, associated with an increased FIRM:CFB ratio in the ileum, gained the most body weight, whereas the fish oil-supplemented diet, which has restored this microbial ratio back to normal, resulted in less body weight gain despite a similar energy intake of fat energy. This suggests that the dysbiotic microbiota and associated weight gain produced from the presence of $n-6$ PUFA could be reversed by the presence of $n-3$ PUFA present in fish oil.

High-fat diets composed of maize oil, a source of $n-6$ PUFA, caused bacterial overgrowth unlike rapeseed diets, which contain both $n$ - 6 and $n$-3 PUFA and oleic acid. Bacterial overgrowth is observed in diseases such as diverticulitis ${ }^{(39)}$, irritable bowel syndrome ${ }^{(40)}$ and IBD $^{(34)}$. Although the exact mechanism by which increased numbers of bacteria affect gut homeostasis is unknown, it may simply be due to the increase in bacterial antigens causing chronic and inappropriate activation of immune responses. In support of this, the high-fat diet composed of maize oil resulted in bacterial infiltration across the intestinal barrier, which was associated with increased infiltration of macrophages and neutrophils. Supplementing the maize oil diet with fish oil led to a reversal of these phenotypes: bacterial load, bacterial composition and inflammatory cell infiltration all returned to the normal chow levels. 



Fig. 5. Fish oil supplementation induces oxidative stress in the ileum in aged mice. Using immunofluorescence and an antibody specific to the oxidative marker 4-hydroxynonenal (4-HNE; red), the fish oil supplemented group shows increased 4-HNE staining in the submucosal region of the ileal. (a) Representative images of ileal sections stained with 4-HNE, where host cell nuclei are stained with 4',6-diamidino-2-phenylindole (DAPI) (blue) (bar $=100 \mu$ m; $200 \times$ magnification). (b) Quantification of 4-HNE fluorescence intensity per tissue section using Metamorphosis software $\left({ }^{\star \star \star} P<0.001\right)$.

Evidence indicates that the intestinal microbiota can induce, alter or even prevent inflammatory diseases such as $\mathrm{IBD}^{(41)}$, the metabolic syndrome ${ }^{(42)}$, diabetes and obesity ${ }^{(43-45)}$. Here, we observed that there was increased infiltration of macrophages and neutrophils in the maize and rapeseed oil diet groups and that fish oil supplementation reversed this infiltration and recruited $T_{\text {reg }}$ cells into the submucosae, which is in turn important for immune suppression. $T_{\text {reg }}$ cells are important during the body's defence against inappropriate immune responses ${ }^{(46)}$ and are induced through stimulation of the immune system by the microbiota ${ }^{(47)}$. This suggests that the $n-3$ PUFA found in fish oil may be able to elicit its anti-

inflammatory properties in the gut by stimulating the recruitment of $\mathrm{T}_{\mathrm{reg}}$ cells.

Fish oil supplementation resulted in elevated oxidative stress in the ileum. 4-HNE is an aldehyde derived specifically from lipid peroxidation of $n$-6 PUFA, like arachidonic acid and linoleic $\operatorname{acid}^{(48)}$. Due to the presence of multiple double bonds, PUFA in general are prone to oxidation. Interestingly, addition of fish oil, rich in long-chain $n-3$ PUFA like DHA (six double bonds) and EPA (five double bonds), to the $n-6$ PUFA-rich diet promoted the generation of the $n-6$ PUFA oxidised byproduct, 4-HNE. Similar results in the liver have been previously reported, where $8 \%(\mathrm{w} / \mathrm{w})$ fish oil augmented oxidative stress biomarkers ${ }^{(29)}$. These results suggest that, while fish oil supplementation can reverse detrimental effects of excess $n$ - 6 PUFA diets, including microbial dysbiosis and inflammatory cell infiltration, the combination of PUFA could also lead to increased oxidative stress in aged mice. Although not determined in the present study, it is possible that fish oil supplementation, while restoring a similar ratio of various phyla to that seen for normal chow, also altered an yet unidentified microbial species within these phyla, contributing to the increased oxidative stress in the fish oil diet group.

\section{Conclusions}

High-fat diets rich in $n$-6 PUFA cause dysbiosis, and induce weight gain and inflammation. Fish oil supplementation reversed these effects, but also resulted in oxidative stress. Since oxidative damage is a detrimental effect associated with ageing, eating diets rich in $n-3$ PUFA with high levels of $n-6$ PUFA, as is recommended to combat excess $n-6$ PUFA in present 'Western' diets, may exacerbate gastrointestinal disorders in a vulnerable aged population.

\section{Acknowledgements}

E. M. was funded by an I.K. Barber School of Arts and Sciences Undergraduate Research Award and D. D. was funded by a Canadian Association of Gastroenterology (CAG) summer-student award. S. G. is a Canadian Diabetes Association (CDA) Scholar funded through CDA and jointly funded with D. L. G. through the Bill and Melinda Gates Foundation. D. L. G. is also funded through the Crohn's and Colitis Foundation of Canada, Natural Sciences and Engineering Research Council and the Intestinal Diseases Education Awareness Society. None of the authors had a conflict of interest to declare. Sources of funding for this project were the Natural Sciences and Engineering Research Council and the Intestinal Diseases Education Awareness Society. The author contributions are as follows: S. G. contributed to the study concept and design; acquisition of data; analysis and interpretation of data; drafting of the manuscript; critical revision of the manuscript for important intellectual content; and study 
supervision; E. M. aided in the acquisition of data; analysis of data; drafting of the manuscript; and statistical analysis; D. D. took part in the acquisition of data; analysis of data; and statistical analysis; C. D. aided in acquisition of data; D. L. G. contributed to the study concept and design; acquisition of data; analysis and interpretation of data; drafting of the manuscript; critical revision of the manuscript for important intellectual content; statistical analysis; obtained funding; administrative, technical or material support; and study supervision.

\section{References}

1. Walter J, Britton RA \& Roos S (2011) Host-microbial symbiosis in the vertebrate gastrointestinal tract and the Lactobacillus reuteri paradigm. Proc Natl Acad Sci U S A $\mathbf{1 0 8}$, Suppl. 1, 4645-4652.

2. Arumugam M, Raes J \& Pelletier E (2011) Enterotypes of the human gut microbiome. Nature 473, 173-180.

3. Korecka A \& Arulampalam V (2012) The gut microbiome: scourge, sentinel or spectator? J Oral Microbiol 4 (Epublication 21 February 2012).

4. Ley R, Hamady M \& Lozupone C (2008) Evolution of mammals and their gut microbes. Science 320, 1647-1651.

5. Ley R, Turnbaugh P, Klein S, et al. (2006) Microbial ecology: human gut microbes assciated with obesity. Nature $\mathbf{4 4 4}$, 1022-1023.

6. Sokol H, Seksik P, Furet JP, et al. (2009) Low counts of Faecalibacterium prausnitzii in colitis microbiota. Inflamm Bowel Dis 15, 1183-1189.

7. Mariat D, Firmesse O, Levenez F, et al. (2009) The Firmicutes/ Bacteroidetes ratio of the human microbiota changes with age. BMC Microbiol 9, 123.

8. Wen L, Ley RE, Volchkov PY, et al. (2008) Innate immunity and intestinal microbiota in the development of type 1 diabetes. Nature 455, 1109-1113.

9. Giongo A, Gano KA, Crabb DB, et al. (2011) Toward defining the autoimmune microbiome for type 1 diabetes. ISMEJ 5, 82-91.

10. Fava F, Gitau R, Griffin BA, et al. (2012) The type and quantity of dietary fat and carbohydrate alter faecal microbiome and short-chain fatty acid excretion in a metabolic syndrome 'at-risk' population. Int J Obes (Lond) (Epublication ahead of print version 13 March 2012)

11. Caricilli A, Picardi P \& de Abreu L (2011) Gut microbiota is a key modulatator of insulin resistance in TLR2 knockout mice. PLoS Biol 9, 1-21.

12. Blasbalg TL, Hibbeln JR, Ramsden CE, et al. (2011) Changes in consumption of omega- 3 and omega- 6 fatty acids in the United States during the 20th century. Am J Clin Nutr 93, 950-962.

13. Tjonneland A, Overvad K, Bergmann MM, et al. (2009) Linoleic acid, a dietary $n-6$ polyunsaturated fatty acid, and the aetiology of ulcerative colitis: a nested case-control study within a European prospective cohort study. Gut 58, 1606-1611

14. Canete R, Gil-Campos M, Aguilera CM, et al. (2007) Development of insulin resistance and its relation to diet in the obese child. Eur J Nutr 46, 181-187.

15. Ramel A, Martinez A, Kiely M, et al. (2008) Beneficial effects of long-chain $n-3$ fatty acids included in an energy-restricted diet on insulin resistance in overweight and obese European young adults. Diabetologia 51, 1261-1268.

16. Reaven P, Parthasarathy S, Grasse BJ, et al. (1991) Feasibility of using an oleate-rich diet to reduce the susceptibility of low-density lipoprotein to oxidative modification in humans. Am J Clin Nutr 54, 701-706.

17. Abbey M, Belling GB, Noakes M, et al. (1993) Oxidation of low-density lipoproteins: intraindividual variability and the effect of dietary linoleate supplementation. Am J Clin Nutr 57, 391-398.

18. Fernandez-Banares F, Esteve M, Navarro E, et al. (1996) Changes of the mucosal $n-3$ and $n-6$ fatty acid status occur early in the colorectal adenoma-carcinoma sequence. Gut 38, 254-259.

19. Nkondjock A, Shatenstein B, Maisonneuve P, et al. (2003) Assessment of risk associated with specific fatty acids and colorectal cancer among French-Canadians in Montreal: a case-control study. Int J Epidemiol 32, 200-209.

20. Nkondjock A, Krewski D, Johnson KC, et al. (2005) Specific fatty acid intake and the risk of pancreatic cancer in Canada. Br J Cancer 92, 971-977.

21. Belluzzi A, Brignola C, Campieri M, et al. (1996) Effect of an enteric-coated fish-oil preparation on relapses in Crohn's disease. $N$ Engl J Med 334, 1557-1560.

22. Innis SM \& Jacobson K (2007) Dietary lipids in early development and intestinal inflammatory disease. Nutr Rev $\mathbf{6 5}$, S188-S193.

23. Dominguez-Bello MG, Blaser MJ, Ley RE, et al. (2011) Development of the human gastrointestinal microbiota and insights from high-throughput sequencing. Gastroenterology 140, 1713-1719.

24. Biagi E, Hylund L, Candela M, et al. (2010) Through ageing, and beyond gut microbiota and inflammatory status in seniors and centenarians. PLoS One 5, e10667.

25. Hill JO, Melanson EL \& Wyatt HT (2000) Dietary fat intake and regulation of energy balance: implications for obesity. J Nutr 130, 284S-288S.

26. Portillo MP, Tueros AI, Perona JS, et al. (1999) Modifications induced by dietary lipid source in adipose tissue phospholipid fatty acids and their consequences in lipid mobilization. Br J Nutr 82, 319-327.

27. Kris-Etherton PM, Harris WS \& Appel LJ (2002) Fish consumption, fish oil, omega-3 fatty acids, and cardiovascular disease. Circulation 106, 2747-2757.

28. Gebauer SK, Psota TL, Harris WS, et al. (2006) n-3 Fatty acid dietary recommendations and food sources to achieve essentiality and cardiovascular benefits. Am J Clin Nutr 83, 1526S-1535S.

29. Hammer CT \& Wills ED (1978) The role of lipid components of the diet in the regulation of the fatty acid composition of the rat liver endoplasmic reticulum and lipid peroxidation. Biochem J 174, 585-593.

30. Yuan YV \& Kitts DD (2003) Dietary ( $n$-3) fat and cholesterol alter tissue antioxidant enzymes and susceptibility to oxidation in SHR and WKY rats. J Nutr 133, 679-688.

31. Ghosh S, Dai C, Brown K, et al. (2011) Colonic microbiota alters host susceptibility to infectious colitis by modulating inflammation, redox status, and ion transporter gene expression. Am J Physiol Gastrointest Liver Physiol 301, G39-G49.

32. Wagner M, Amann R, Lemmer H, et al. (1993) Probing activated sludge with oligonucleotides specific for proteobacteria: inadequacy of culture-dependent methods for describing microbial community structure. Appl Environ Microbiol 59, 1520-1525.

33. Meier H, Amann R, Ludwig W, et al. (1999) Specific oligonucleotide probes for in situ detection of a major group of gram-positive bacteria with low DNA G + C content. Syst Appl Microbiol 22, 186-196. 
34. Klaus J, Spaniol U, Adler G, et al. (2009) Small intestinal bacterial overgrowth mimicking acute flare as a pitfall in patients with Crohn's disease. BMC Gastroenterol 9, 61.

35. Murphy EF, Cotter PD, Healy S, et al. (2010) Composition and energy harvesting capacity of the gut microbiota: relationship to diet, obesity and time in mouse models. Gut 59, 1635-1642.

36. Statistics C (2006) Food Statistics 2005. http://www.statcan. gc.ca/pub/21-020-x/21-020-x2005002-eng.pdf

37. Goyens PL, Spilker ME, Zock PL, et al. (2006) Conversion of alpha-linolenic acid in humans is influenced by the absolute amounts of alpha-linolenic acid and linoleic acid in the diet and not by their ratio. Am J Clin Nutr 84, 44-53.

38. Turnbaugh PJ, Ley RE, Mahowald MA, et al. (2006) An obesity-associated gut microbiome with increased capacity for energy harvest. Nature 444, 1027-1031.

39. Tursi A, Brandimarte G, Giorgetti GM, et al. (2005) Assessment of small intestinal bacterial overgrowth in uncomplicated acute diverticulitis of the colon. World $J$ Gastroenterol 11, 2773-2776.

40. Pimentel M, Chow EJ \& Lin HC (2000) Eradication of small intestinal bacterial overgrowth reduces symptoms of irritable bowel syndrome. Am J Gastroenterol 95, 3503-3506.

41. Packey CD \& Sartor RB (2009) Commensal bacteria, traditional and opportunistic pathogens, dysbiosis and bacterial killing in inflammatory bowel diseases. Curr Opin Infect Dis 22, 292-301.

42. Vijay-Kumar M, Aitken JD, Carvalho FA, et al. (2010) Metabolic syndrome and altered gut microbiota in mice lacking Toll-like receptor 5. Science 328, 228-231.

43. Cani PD, Delzenne NM, Amar J, et al. (2008) Role of gut microflora in the development of obesity and insulin resistance following high-fat diet feeding. Pathol Biol (Paris) 56, 305-309.

44. Turnbaugh PJ, Backhed F, Fulton L, et al. (2008) Dietinduced obesity is linked to marked but reversible alterations in the mouse distal gut microbiome. Cell Host Microbe $\mathbf{3}$ 213-223.

45. Tilg H, Moschen AR \& Kaser A (2009) Obesity and the microbiota. Gastroenterology 136, 1476-1483.

46. Atarashi K, Tanoue T, Shima T, et al. (2011) Induction of colonic regulatory $\mathrm{T}$ cells by indigenous Clostridium species. Science 331, 337-341.

47. Geuking MB, Cahenzli J, Lawson MA, et al. (2011) Intestinal bacterial colonization induces mutualistic regulatory $\mathrm{T}$ cell responses. Immunity 34, 794-806.

48. Esterbauer H, Schaur RJ \& Zollner H (1991) Chemistry and biochemistry of 4-hydroxynonenal, malonaldehyde and related aldehydes. Free Radic Biol Med 11, 81-128. 